\title{
Estudio de la ocupación del tiempo libre de los escolares Study of the participation of schoolchildren in leisuretime
}

\author{
*Yolanda Hermoso Vega, *Ventura Garccía Preciados, **Jose Luís Chinchilla Minguet \\ *Universidad de Extremadura, ${ }^{* *}$ Universidad de Málaga
}

Resumen: El objetivo de este trabajo es validar el cuestionario creado para analizar la ocupación del tiempo libre de jóvenes y adolescentes, a través de la participación o no en actividades extraescolares una vez finalizada la jornada lectiva. En Extremadura, existe la posibilidad de elegir la modalidad de jornada escolar con actividades formativas complementarias (AFC) que comprende actividades lectivas en horario de mañana y actividades formativas complementarias en horario de tarde. En consecuencia, se podría afirmar que los escolares han visto incrementado su tiempo libre. En el estudio han participado escolares $(n=773$ ) del tercer ciclo de educación primaria y de educación secundaria de diferentes centros públicos y concertados de Badajoz y Poblados. Se llevó a cabo un proceso de construcción, pilotaje y validación del cuestionario a través del análisis estadístico de los ítems, sometido a valoración por un grupo de expertos y prueba por el grupo experimental, cumpliéndose los requisitos de validez externa, expresados en la representatividad de la muestra de la población objeto de estudio, validez interna equivalente al concepto de calidad de encuesta y validez de contenido expresado en el correcto diseño y elaboración del cuestionario definitivo.

Palabra clave: Tiempo libre, ocio, actividades extraescolares.

Abstract: The objective of this work is to validate the questionnaire that was produced to analyze the use of leisure time by youths and adolescents, by participating or not, in extra-curricular activities at the end of the school day. In Extremadura, it is possible to elect the shape of the school day with formative complimentary activities (DOE, 2002) which include school activities during the morning timetable and formative complimentary activities during the afternoon timetable. And, as a consequence it has been proved that school children have seen their leisure time increase. In this study, 773 school children have taken part, both from the third year of primary school and the compulsory secondary school from different state schools in Badajoz and the provinces. The construction, piloting and validation of the questionnaire was carried out by means of the statistical analysis of the items, which was assessed by a group of experts and tested by the experimental group. The necessary requisites were fulfilled as follows; external validation expressed in the representative sample of the population which were the object of this study, internal validation equivalent to the concept of quality of the questionnaire and content validation manifest in the correct design and elaboration of the definitive questionnaire.

Key words: Leisure time, leisure, out of school activities.

\section{Introducción}

El tiempo fuera del horario lectivo, surgido en la jornada escolar continua, supone un auténtico desafío para los padres y madres que necesitan conciliar los horarios laborales y familiares. Esta creciente situación denecesidad de utilización de recursos extrafamiliares públicos hallevado a la Junta de Extremadura (una vez asumidas las competencias en materia de educación no universitaria en el curso académico 2000-01) a la elección por parte de los centros que así lo deseen de la jornada escolar con AFC, que comprende actividades lectivas en horario de mañana y AFC en horario de tarde. La ocupación del tiempo libre en actividades extraescolares organizadas se presenta como una potencial fuente de beneficios, y por otra parte, los padres muestran gran preocupación por la educación que puedan recibir sus hijos e hijas para la vida, hecho que influye en las alternativas que escogen para dichas actividades.

La cercanía y la actualidad del proyecto de AFC que se desarrolla en la comunidad autónoma de Extremadura a través de los centros docentes, unido a la falta de proyectos idénticos en otras comunidades autónomas, pueden explicar la escasez de investigaciones. Decualquiermodo, existen algunos, sobre las actividades educativas fuera del horario lectivo (PérezDíaz, Rodríguez y Sánchez, 2001; Instituto Nacional de Calidad y Evaluación, 2001; Trilla y García, 2004; Trilla y Ríos, 2005; Fundación Jaume Bofill, 2006, 2009; Molinuevo, 2008, 2009).

Obras de autores como Hernández y Velázquez (1996) y Santos y Sicilia (1998) hacen referencia a las actividades físicas extraescolares en los centros educativos y la legislación que los regula. Entre los estudios más específicos, encontramos algunos que relacionan los hábitos de vida de jóvenes y adolescentes y las actividades extraescolares (Castillo y Balaguer, 2001; Hidalgo, 2005; Varela, 2006; Mollá, 2007; Luengo, 2007); la ocupación de tiempo libre y el estilo de vida saludable entre los

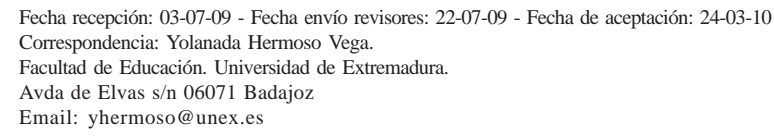

jóvenes (Hernándezy Velázquez, 2007; Nuviala, Munguía, Fernández, Ruiz y García, 2009).

También hemos encontrado numerosos trabajos que aluden a la participación de jóvenes y adolescentes en actividades físico-deportiva fuera del horario escolar (Mendoza Sagrera y Batista 1994; Casimiro, 1999; Cantera, 2000; Guerrero, 2000; Sallis, 2000; García Ferrando, 2001, 2006; Chillón, Tercedor, Delgado, González-Gross, 2002; Gavarry, Giacomoni, Bernard, Seymat, and Falgairette, 2003; Nuviala, Ruiz y García , 2003; Tercedor et al., 2003; Chillón, 2005; Hernández y Velázquez, 2007)

Destacamos asimismo algunas investigaciones que relacionan el posible papel de las actividades extraescolares en la promoción del rendimiento académico (Ponce de León, 1998; Eccles and Templeton 2002; Trilla y Ríos, 2005; Molinuevo, 2008).

Nuestro trabajo es el resultado de la reflexión iniciada con la jornada escolar continua, que supone un incremento del tiempo libre de los escolares. La utilización adecuada de este tiempo libre podría contribuir a paliar problemas graves que afectan a los escolares, como la obesidad asociada al descenso detectado en los niveles de actividad físicay deportiva en adolescentes a edades cada vez más tempranas o la violencia en los centros educativos. En esta línea, los requisitos exigidos por la Junta de Extremadura (que financia el programa de AFC) para la selección y contratación de los profesionales encargados del desarrollo de las actividades revelan la orientación que se pretende otorgar a las actividades extraescolares, ya que se concede prioridad a las titulaciones de EF y deportiva, de mayor bagaje pedagógico que permitan un adecuado y correcto desarrollo de las actividades físico-deportivas extraescolares.

\section{Material y Métodos}

Nuestra población objeto de estudio está compuesta por el alumnado que cursa el último ciclo de Primaria ( $5^{\circ}$ y $6^{\circ}$ de primaria) y toda la enseñanza secundaria obligatoria $\left(1^{\circ}, 2^{\circ}, 3^{\circ}\right.$ y $\left.4^{\circ} \mathrm{ESO}\right)$, en los diferentes centros públicos y privados concertados de Badajoz y sus Poblados. El estudio se ha centrado en escolares con un rango de edad comprendido entre los 10-16 años. 
Se seleccionó la edad mínima de 10 años, como comienzo del último ciclo de Primaria, donde los escolares, tienen adquirido una comprensión lectora adecuada al nivel de las encuestas administradas y donde el proceso de adscripción de la nueva jornada escolar de los colegios de educación infantil y primaria contempla un proyecto de AFC desde las 16.00 a las 18.00 horas.

Por otro parte, los escolares de los centros públicos de la comunidad autónoma de Extremadura, a partir de los 12 años, cuando finalizan el último ciclo deprimaria cambian de centropara iniciar la secundaria obligatoria a los institutos de enseñanza secundaria (IES), que por zonas le corresponden al colegio público al que se encuentran adscritos, sin embargo el proyecto de AFC no se desarrolla en los IES.

La justificación para seleccionar $4^{\circ}$ de la ESO como edad máxima de nuestra población de estudio viene determinada, por ser la finalización de los estudios obligatorios. Y en términos generales indicar que las etapas educativas elegidas se pueden considerar comouna fase de la vida donde los hábitos relacionados con la ocupación del tiempo libre comienzan a afianzarse, positiva o negativamente, siendo fundamentales para organizar un tiempo libre de calidad en un futuro, ya sea en el ámbito de las actividades físicodeportivas como en el ámbito de las actividades culturales y o artísticas.

La población total (universo) en el curso 2007-08, sumando el alumnado de los centros públicos y concertados de Badajoz y Poblados, asciendeen $3^{\circ}$ ciclodeprimariaa 3563 alumnos/as, en $1^{\circ}$ ciclodesecundaria a 3918 alumnos/as y en $2^{\circ}$ ciclo de secundaria a 3617 alumnos/as, siendo la suma de los tres ciclos el universo de nuestro estudio con 11.061 alumnos/as.

\subsection{Muestra}

En el estudio han participado escolares $(\mathrm{n}=776$ ) del tercer ciclo de primaria y de la secundaria obligatoria de diferentes centros públicos y concertados de Badajoz y Poblados. El diseño muestral utilizado fue el bietápico estratificado con submuestreo. La unidad primaria demuestreo fue el centro educativo (conglomerados), mientras que la unidad secundaria o elemental fue el alumno o alumna perteneciente al centro educativo seleccionado. La selección de las unidades muestrales fue aleatoria simple. Las variables de estratificación de la muestra fueron: zona (9 zonas definidas en base a la delegación provincial de educación de Badajoz), ciclo educativo ( $3^{\text {er }}$ ciclo de primaria, ${ }^{\text {er }}$ ciclo de secundaria y $2^{\circ}$ ciclo de secundaria) y sexo. La asignación de la muestra fue proporcional al tamaño poblacional en cada variable de estratificación

El tamaño muestral total de ambos géneros asciende a 387 alumnos y 389 alumnas, cuyas edades oscilan entre los 10 y 16 años. Esto supone una validez en los resultados con un margen de error muestral de $\pm 3 \%$ y un error de confianza del $95 \%$.

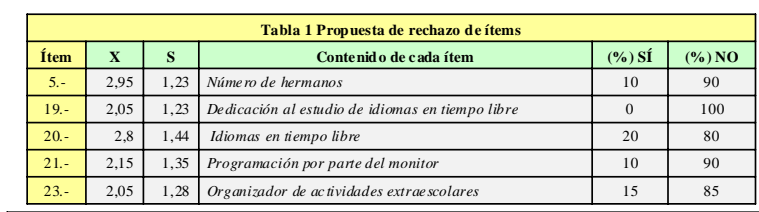

\begin{tabular}{|c|c|c|c|c|c|c|c|c|c|}
\hline \multicolumn{10}{|c|}{$\begin{array}{l}\text { Tabla 3. Participación en actividades extraescolares } \\
\text { en relación al sexo, la edad y el tipo de centro }\end{array}$} \\
\hline & \multicolumn{2}{|c|}{ SEXO**** } & \multicolumn{3}{|c|}{ EDAD (Ciclo)**** } & \multicolumn{3}{|c|}{ TIPO DE CENTRO } & \multirow{2}{*}{ TOT AL } \\
\hline & Hombres & M ujeres & $\mathrm{P}_{3}$ & $s_{1}$ & $\mathrm{~S}_{2}$ & Concertado & Público & Privado & \\
\hline sí & 94,90 & 74,0 & 95,6 & 80,90 & 75,60 & 87,20 & 82,30 & 86,70 & 84,20 \\
\hline No & 5,60 & 26,0 & 15,80 & 4,40 & 19,10 & 12,80 & 17,70 & 13,30 & 15,80 \\
\hline
\end{tabular}

\begin{tabular}{|c|c|c|c|c|c|c|c|}
\hline \multicolumn{8}{|c|}{$\begin{array}{l}\text { Tabla 4. Participación en actividades extraescolares } \\
\text { en relación al nivel de e studios del padre y la mad re }\end{array}$} \\
\hline & \multicolumn{3}{|c|}{ PADRE**** } & \multicolumn{3}{|c|}{ MADRE $* * *$} & \multirow[b]{2}{*}{ TOTAL } \\
\hline & $\begin{array}{l}\text { Grad. Es. } \\
\text { Sin estud }\end{array}$ & $\begin{array}{l}\text { Estudios } \\
\text { Medios }\end{array}$ & $\begin{array}{l}\text { Estudios } \\
\text { Superiores }\end{array}$ & $\begin{array}{l}\text { Grad. Es. } \\
\text { Sin estudd }\end{array}$ & $\begin{array}{l}\text { Estudios } \\
\text { Medios }\end{array}$ & $\begin{array}{l}\text { Estudios } \\
\text { Superiores }\end{array}$ & \\
\hline sí & 79,50 & 81,60 & 95,80 & 79,0 & 85,0 & 94,10 & 84,20 \\
\hline No & 20,50 & 18,40 & 4,20 & 21,0 & 15,0 & 5,90 & 15,80 \\
\hline
\end{tabular}

\subsection{Instrumentos}

Lafundamentaciónmetodológica delainvestigación objetodeestudio está basado en la metodología de encuestas según Fontes de Gracia, García, Garriga, Pérez-Llantada, Sarriá(2007), para ello definimosnuestra población objeto de estudio, determinamos la muestra representativa, diseñamos el tipo de muestreo utilizado, establecemos la elección y construcción de los instrumentos de recogida de información, a través del pilotaje y validación de un cuestionario, realizamos la codificación y ordenación de los datos, para finalizar con el análisis de los resultados e informe final.

La elaboración y el diseño del cuestionario objeto de nuestra investigación tiene como principal objetivo conocer la ocupación del tiempo libre de los escolares, una vez finalizada la jornada lectiva, en edades comprendidas entre los 10-16 años. La formulación de preguntas y respuestas, a través de la encuesta, se ha utilizado con el fin de obtener datos descriptivos, que los sujetos encuestados puedan proporcionar a partir de su propia experiencia. No medimos variable alguna, solo valoramos una serie de actitudes y opiniones de los escolares en ese tramo de edad. El lenguaje utilizado se ha adaptado al nivel de los estudiantes del último ciclo de primaria y delos estudiantes de secundaria, de los centros de Badajoz y Poblados donde se ha realizado la investigación, para facilitar su comprensión.

Las cuestiones tuvieron distinta procedencia: algunas fueron tomadas literalmente de cuestionarios con fines similares (García Ferrando, 1991, 1993, 1996, 2001, 2006; González Pajuelo, 1999; García Preciados 2003 y encuestas CIS -Centro de Investigaciones Sociológicas-, 2000), otros fueron reformuladas para que respondieran a los propósitos del cuestionario y otros los redactamos específicamente para la ocasión. Una vez determinados losítems dela versión experimental se procedió a determinar la estructura del cuestionario, que tras las conclusiones obtenidas en el estudio piloto y en el grupo de expertos, se decidió por el tipo de pregunta cerrada -de elección simple o múltiple-, debido a la edad de los sujetos objeto de estudio, para evitar al máximo las incertidumbres y que se dejen respuestas sin contestar, además de considerar la rapidezy la ausencia de fatiga de los escolares como razón fundamental para la elección de éste tipo de cuestionario.

Las preguntas se han ordenado por bloques: un bloque de datos identificativos o de control, donde se recogen datos sociodemográficos del encuestado/a y datos de sus familiares, como son el sexo, la edad, las condiciones laborales de la unidad familiar, nivel socio-cultural de los padres/madres, zona de la vivienda familiar. Una segunda parte de elaboración de preguntas subdivididas en 4 bloques, en función del tipo de actividad extraescolar organizada o no organizada que los escolares realizan una vez finalizadas las actividades lectivas en el centro educativo: Bloque I: realización de actividades extraescolares (tipo, motivos, 
frecuencia, lugar, utilidad), actividades físico-deportivas y de competición, actividades culturales y/o artísticas, idiomas, satisfacción uso tiempo libre. Bloque II: realización de actividades organizadas (programación, organizador, elección de actividades y actividades no realizadas). Bloque III: realización de actividades no organizadas (motivos). Bloque IV: realización de otras actividades (dedicación tiempo libre, motivos no actividad en tiempo libre). Se le han pasado las correspondientes pruebas de validez de contenido, validez externa y validez interna a través del estudio piloto y de las diferentes aportaciones hechas por los expertos que han colaborado en la investigación.

Una vez realizado el cuestionario en su versión experimental, siguiendo las recomendaciones citadas, fue sometido a la valoración del grupo de expertos para cubrir los requisitos exigidos en todo proceso de investigación. Martín Arribas (2004) informa que la validez de contenido tiene por objetivo conocer si el cuestionario elaborado, y por tanto los ítems elegidos son indicadores de lo que se pretende medir. Para ello, se trata de someter el cuestionario a la valoración de investigadores y expertos que deben juzgar la capacidad de éste para evaluar todas las dimensiones que deseamos medir.

El análisis delavaloración general sobrela adecuación del cuestionario presentado al grupo de expertos se realizó a través de una escala de puntuación de Likert de 1 a 5 (1=en total desacuerdo, 2=en desacuerdo, $3=$ indiferente, $4=$ de acuerdo y $5=$ =totalmente de acuerdo). Los resultados de dicha valoración arrojaron resultados muy satisfactorios expresados a través de la $(X=$ media y $S=$ desviación estándar $)$ : valoración global del cuestionario: $(X=4,35$ y $S=0,49)$. Estructura del cuestionario: $(X=4,28$ $y S=0,54)$. Compresión de las preguntas: $(X=4,36 y S=0,54)$. Sencillez y claridad: $(X=4,43$ y $S=0,56)$.

Con objeto de cumplir el criterio de validez interna equivalente al concepto de calidad de las encuesta, se procedió al análisis de la valoración de los grupos de expertos a cada uno de los ítems, sobre los modelos elaborados al efecto que consistieron en unas plantillas de recogida de información.

Según las medias observadas en cada uno de los ítems, podemos afirmar que en términos generales las variables que se pretenden medir se distribuyen normalmente. A tal conclusión llegamos atendiendo a las desviaciones típicas, que en la mayoría de los casos, se sitúan en torno a los valores establecidos para una distribución normal (--+/-2z). Del mismo modo hemos de señalar que aquellas variables cuya desviación típica se alejan de la distribución normal reflejados en la (tabla 1), nos indican que debemos considerar su rechazo para la confección del cuestionario definitivo.

Una vez analizados todos los datos obtenidos y cumplidos los criterios de validez externa expresados en la representatividad de la muestra de la población objeto de estudio; validez interna respecto a la calidad de las encuestas y validez de contenido expresado en el correcto diseño y elaboración del cuestionario, se procedió a la reelaboración del cuestionario definitivo y se establecieron los procedimientos para su aplicación.

Las variables que formaron parte del cuestionario definitivo, tras el análisis expuesto quedan reflejadas en la (tabla 2). El cuestionario fue «autoadministrado» (Hernández, Fernández y Baptista, 2006) a los alumnos/as por la investigadora principal, seentregaron de forma colectiva $\mathrm{y}$ tras un breve periodo de tiempo se recogen personalmente. La elaboración del mismo ha sido costosa por la dificultad de elaborar preguntas y respuestas que cubran todos los objetivos y respuestas posibles y por la exigencia metódica que supone el proceso sistemático deelaboracióny validación deun cuestionario con una secuencia ordenada de las diferentes etapas.

\subsection{Análisis de datos}

Una vez finalizada la etapa de recogida de datos, se procedió a su codificación a través de lectura óptica que permite la introducción de datos de una forma muy rápida y fiable (Fontes de Gracia et al, 2007). Tras la recogida de la información, ésta fue procesada en la hoja de cálculo Excel de Microsoft Excel siendo posteriormente exportada a SPSS 14.0 para la codificación, etiquetado, depuración y análisis de los datos.
El análisis estadístico de los datos que se presenta en este informe ha sido descriptivo obteniendo estimaciones porcentuales sobre las variables de estudio y bivariante mediante el estadístico chi-cuadrado de Pearson y los residuos tipificados corregidos, para estudiar la asociación de las variables de estudio con el sexo, ciclo educativo, tipo de centro, zona, ocupación y nivel de estudios de los padres de los escolares pacenses. También se utilizó el estadístico chi-cuadrado de Mantel y Haenszel para contrastar la linealidad entre las variables de estudio con el ciclo formativo o con el nivel de estudios de los padres y madres. Con el fin de corregir las desviaciones de la muestra final con respecto a la población de estudio se calcularon factores de corrección según zona, ciclo educativo y sexo (mínimo 0,81; máximo 1,18). En todo el análisis se consideró significación con valores de $\mathrm{p}<=0,05$ y casi significación con $0,05<\mathrm{p}<=0,1$.

\section{Resultados}

Los escolares pacenses presentan un índice de participación muy alto en la realización de actividades fuera del horario lectivo. Un 84,20\% manifiestan realizar actividades extraescolares en su tiempo libre, significativamente superior al 15,80\% que manifiesta no realizar ningún tipo de actividad extraescolar (tabla 3). Si analizamos la realización de actividades extraescolares según sexo, edad y tipo de centro, observaremos diferencias significativas respecto a la variable sexo y edad, y ligeras diferencias respecto a la variable tipo de centro:

En relación al sexo (tabla 3 y figura 1 ) se puede observar que es significativamente menor la realización de actividades extraescolares en chicas que en chicos (74,0\% y $94,40 \%$ respectivamente). Respecto a la edad también hay importantes diferencias: un 95,6\% de los escolares de tercer ciclo de primaria realiza actividades extraescolares, porcentaje significativamente superior al observado en primer y segundo ciclo de secundaria (80,9\% y 75,6\% respectivamente). Se observa una tendencia de disminución en el porcentaje de participación en actividades extraescolares en relación a la edad (figura 2). La participación en actividades extraescolares por tipo de centro (tabla 3) presenta ligeras diferencias, un 82,3\% de la población escolar pacense realiza actividades extraescolares en centros públicos, siendo ligeramente inferior al 87,2\% de la población pacense que realiza actividades extraescolares en los centros concertados (figura 3)

Respecto al nivel de estudios de los padres y madres, la participación de los escolares en actividades extraescolares disminuye de manera muy significativa conforme disminuye el nivel de estudios de padres y/o madres. Aquellos escolares con padres o madres con estudios universitarios presentan el porcentajemás alto derealización de actividades extraescolares con un 95,80\% y 94,10\% (padrey madre respectivamente) frente al 79,50\% y 79,0\% de adolescentes con padres o madres con nivel de estudios más bajos (tabla 4 y figuras 4.a y 4.b).

$\mathrm{Si}$ analizamos el tipo de actividad que realizan los escolares en su tiempo libre, las actividades que más predominan son las físico-deportivas (organizadas y no organizadas), 58,90\% y 52,90\% respectivamente y
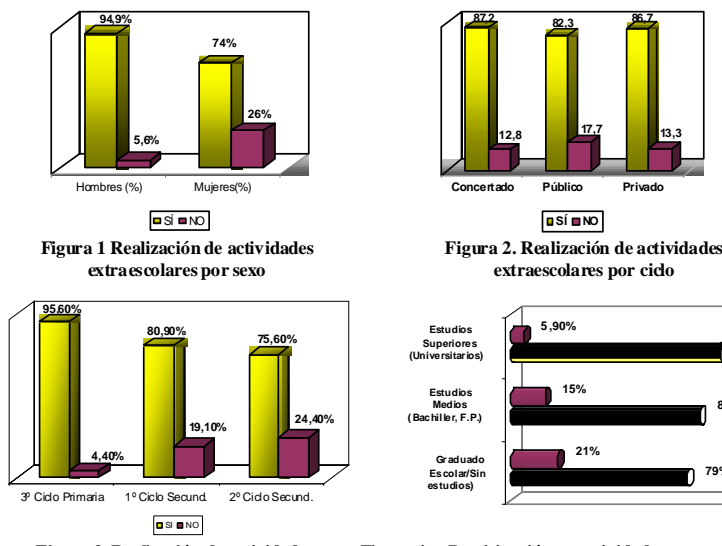

(1) Figura 3. Realización de actividades extraescolares por tipo de centro

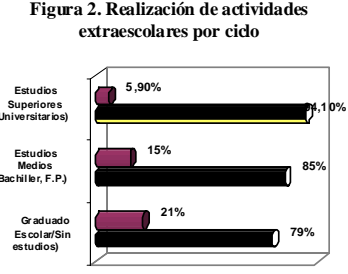

Figura 4.a. Participaín en actividades extaescoles en relación al nivel de estudios del padre 

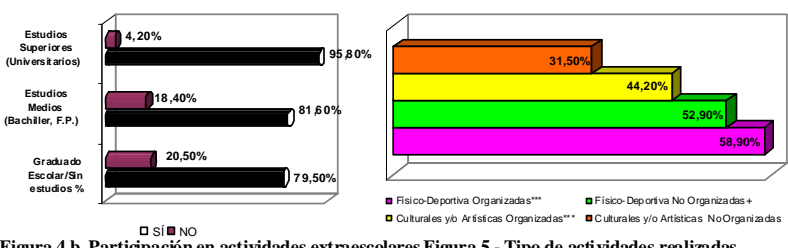

Figura 4.b. Participación en actividades extraescolares Figura 5.- Tipo de actividades realizadas en relación al nivel de estudios de la madre.
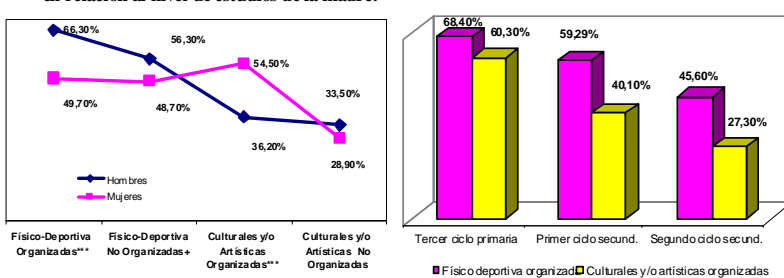

Figura 6.- Tipo de actividades por sexo

Figura 7.- Tipo de actividades organizadas por ciclo

las que menos predominan son las de tipo culturales y/o artísticas (organizadas y no organizadas) 44,29\% y 31,50\% respectivamente (figura5).

En relación al sexo se puede observar que un 66,3\% de los chicos que realizan actividades extraescolares, son de tipo físico-deportivas organizadas significativamente superior al 49,7\% de las chicas (figura 6) y a mayor edad, menor participación (68,4\% en $3^{\circ}$ ciclo de Primaria, 59,29\% en el primer ciclo de Secundaria y 45,6\% en el segundo ciclo de Secundaria). Se observa una tendencia lineal descendente significativa en el porcentaje de realización de actividades extraescolares físico-deportivas organizadas conforme aumenta el ciclo educativo (figura 7).

\section{Discusión}

La participación de los escolares en actividades extraescolares de nuestro estudio presenta similitudes con el estudio realizado por el ayuntamiento de Barcelona (Fundació Jaume Bofill, 2006) sobre la participación de jóvenes y adolescentes en actividades fuera del horario escolar. En el estudio de la población escolar barcelonesa la participación es del $77,8 \%$, ligeramente menor a la de nuestro estudio. Hay que considerar que el rango de edad del estudio de esta población es de 6-12 años. En relación al sexo el nivel de realización de actividades fuera del horario lectivo observa pocas diferencias entre chicas y chicos (77,1\% y $79 \%$ respectivamente), ello se justifica por la diferencia del rango de edad de la muestra. Estos resultados pueden reflejar la influencia de la edad enlaparticipación en actividades extraescolares. El Instituto Nacional de Calidad y Evaluación (INCE), (2001) informa que los chicos realizan en porcentaje más alto que las chicas actividades extraescolares de forma organizada.

El estudio de la población barcelonesa, destaca la influencia de la edad de forma notable, los escolares de 6-11 años son los que hacen más actividad. También se observa una ligera disminución en la participación a partir de los 11, 12 años (cuando se inicia la educación secundaria obligatoria). (Fundación Jaume Bofill, 2009).

Según el INCE (2001), las actividades extraescolares son realizadas proporcionalmente por menos alumnos de la enseñanza secundaria obligatoria que de la educación primaria. En total, de cada 10 alumnos, uno no realiza ninguna de estas actividades en educación primaria frente a dos alumnos en educación secundaria obligatoria. Hoy en día, estas actividades tienen una fuerte presencia en el tiempo no lectivo en niños y niñas de primaria (Trilla y García, 2004).

En el estudio de Nuviala, Ruiz y García (2003), en la población escolar de la Ribera baja del Ebro con el mismo tramo de edad de nuestro estudio entre 10 y 16 años, la participación del alumnado en actividades físico-deportivas extraescolares organizadas es ligeramente inferior (50,4\%) a la obtenida en nuestro estudio. También encuentran una relación estadísticamente significativa entre el sexo y la edad: las chicas se inscriben menos $(39,1 \%)$ que los chicos $(62,3 \%)$ y a mayor edad, menor participación (58,6\% en tercer ciclo de primaria, 52,7\% en el primer ciclo de secundaria y 42,5\% en el segundo ciclo de secundaria), sin embargo, son ligeramente inferiores a los obtenidos en la ciudad de Badajoz y poblados en el mismo tramo de edad.

En los últimos años, en nuestro país han ido en aumento los porcentajes de participación de niños y niñas de primaria y secundaria en actividades extraescolares. Pérez-Díaz; Rodríguez y Sánchez,(2001) apuntaban que el $69 \%$ de padres de niños y niñas de primaria y secundaria afirmaba quesushijosehijas realizaban al menosuna actividad extraescolar cada semana. Trilla y Ríos (2005) señalaban que el 77,5\% de escolares de primaria practicaba alguna actividad extraescolar durante el curso académico.

La participación en actividades extraescolares por tipo de centro presenta en nuestro estudio ligeras diferencias. Los resultados del estudio de la población escolar barcelonesa (Fundación Jaume Bofill, 2009) son muy similares, aunque con participación ligeramente inferior en los centros concertados y públicos (80,7\% y 75,6\% respectivamente). Según el INCE (2001), de forma general, los alumnos de centros privados participan en actividades extraescolares en mayor proporción que los alumnos de los centros públicos.

Respecto al nivel de estudios de los padres y madres, la participación de los escolares en actividades extraescolares disminuye de manera muy significativa conforme disminuye el nivel de estudios de padres y/o madres. En el estudio realizado por la Fundació Jaume Bofill (2009), los hijos de padres y madres con estudios universitarios tienen una participación superior en actividades extraescolares y de forma similar, según los datos del INCE (2001), la participación en actividades extraescolares está asociada al nivel máximo de estudios de los padres; a mayor nivel de estudios es mayor la proporción de alumnos que realizan más de una actividad extraescolar.

El desarrollo del proyecto de AFC presenta similitudes con las actividades extraescolares expuestas por Gilman, Meyers y Pérez(2004), como actividades constructivas y recomendables para ocupar parte del tiempo fuera del horario lectivo escolar, siendo potencialmente beneficiosas por las características de voluntariedad, estructuración y organización, esfuerzo y desafío, redes de apoyo/capital social y desarrollo de habilidades y competencias.

Hay estudios como los de Dunn, Kinney y Hofferth (2003), que de forma cualitativa analizaron las expectativas de los padres respecto a las actividades extraescolares, éstos las concebían como herramientas útiles para el desarrollo moral, personal y social de los niños/as. Por otra parte, además de proporcionar oportunidades para divertirse e interactuar con otros niños/as fuera del colegio, los padres veían que también les podían proporcionar oportunidades para descubrir talentos innatos y ser físicamente activos.

En la actualidad, se puede afirmar que han aumentado los recursos públicos y privados dirigidos a cubrir las necesidades de cuidado infantojuvenil, para conciliar los horarios laborales y extraescolares como consecuencia de la jornada intensiva, paralelamente los padres actuales se muestran interesados en la formación de sus hijos, hecho que influye en las alternativas que escogen para ocupar el tiempo fuera del horario escolar (Pérez-Díaz, Rodríguez y Sánchez, 2001).

\section{Conclusiones}

A través del cuestionario creado para analizar la ocupación del tiempo libre de jóvenes y adolescentes, donde pretendemos conocer el carácter dinámico o estático de las actividades más habituales que desarrollan en su tiempo libre los escolares pacenses (Badajozy Poblados) con edades comprendidas entre los 10-16 años, podemos concluir que el índice de participación en actividades extraescolares es muy alto, con diferencias significativas respecto al sexo, la edad y el nivel de estudios de padres y madres.

La participación tanto en actividades físico-deportivas como culturales y/o artísticas, está condicionada por la edad. Los alumnos de nuestro estudio más jóvenes (10-12 años) son los más activos. Queremos destacar la importancia y necesidad para promover la actividad física durante períodos específicos de crecimiento, ante el descenso de este tipo de actividad en el período de transición de la primaria a secundaria (12-16 años), con el objeto de prevenir el desarrollo de un estilo de vida inactivo. 
En cuestión de género, se observa una menor participación en actividades físico-deportivas de las chicas frente a la participación de los chicos. La población escolar femenina no se siente atraída hacia deportes de competición, donde predominan valores considerados «masculinos» (competición, fuerza, potencia, éxito, etc.), sin embargo presentan un porcentajemás alto de participación de actividades extraescolares cuando éstas son culturales y/o artísticas.

La participación en actividades extraescolares presenta resultados muy similares en relación a la titularidad de los centros tanto públicos como concertados, ya que el proyecto de AFC se desarrolla en todos los centros de Extremadura que así lo soliciten. Todos los centros de enseñanza públicos y concertados de la ciudad de Badajoz, donde ha sido realizado el estudio, están adscritos al tipo de jornada única. Los hijos/as de padres y madres con estudios universitarios presentan el porcentaje más alto de realización de actividades extraescolares.

La elevada participación de los escolares en el proyecto de AFC en los centros de Extremadura, unida a los altos niveles de satisfacción expresados por el alumnado, revelan la buena acogida que ha tenido este proyecto, que favorece la educación integral de la población escolar, por el desarrollo de una serie de habilidades y competencias y por la utilización saludable de su tiempo libre, sin discriminación alguna por el carácter voluntario y gratuito de las mismas.

La implantación de la nueva jornada escolar en los centros y la posibilidad de ofertar una amplia y variada oferta de actividades extraescolares ha permitido una nueva concepción del tiempo libre en los escolares extremeños, con especial incidencia en las actividades físicas y deportivas que han visto aumentadas el número de horas de práctica motriz.

\section{Bibliografía}

Cantera, M.A. y Devís, J. (2000). Physical activity levels of secondary schoo spanish adolescents. European Journal of Physical Education, 5, 28-44.

Castillo, I., Balaguer, I.(1998). Patrones de actividad físicas en niños y adolescentes. Apunts de Educación Física y Deportes, 54, 22-28.

Castillo, I. y Balaguer, I. (2001). Dimensiones de los motivos de práctica deportivas de los adolescentes valencianos escolarizados. Apunts de Educación Física y Deportes, 63, 22-29.

Casimiro, A.J. (1999). Comparación, evolución y relación de hábitos

saludables y nivel de condición física-salud en escolares, entre final de educación primaria (12 años) y final de educación secundaria obligatoria (16 años). Tesis doctoral. Universidad de Granada.

Casimiro, A. J. (2000a). ¿Se está robotizando el tiempo libre de nuestros jóvenes? Recuperado el 13 de Septiembre de 2007, de http://www.efdeportes.com

Chillón, P., Tercedor, P., Delgado, M. y González-Gross (2002). Actividad f í s i c o deportiva en escolares adolescentes. Retos. Nuevas Tendencias $e$ Educación Física, Deporte y Recreación; 3, 5-12.

Chillón, P. (2005). Efectos de un programa de intervención de Educación Física para la salud en adolescentes de $3^{\circ}$ de ESO. Tesis Doctoral. Universidad de Granada.

Dunne, J.S., Kinney, D.A. y Hofferth, S.L. (2003). Parental ideologies and children's after-school activities. American Behavioral Scientist, 46(10), 1359-1386.

Eccles, J.S., Barber, B.L., Stone, M. y Hunt, J. (2003). Extracurricular Activities and adolescent development. Journal of Social Issue, 59(4), 865-889.

Eccles, J.S.y Templeton, J. (2002). Chapter 4: extracurricular and other after-school activities for youth. Review of research in education, 26, 113-180.

Feldman, A.F. y Matjasko, J.L. (2005). The role of school-based extracurricular activities in adolescent development: a comprehensive review and future directions. Review of Educational Research, 75(2), 159-210.

Feu, S. (2000) La iniciación deportiva de las escuelas deportivas a través de las actividades extraescolares en los Centros Educativos de Primaria. En revista Educaçao Física, vol. IV. N 2, pp. 27-38. vol. IV, nº2, julio-diciembre. APEF de Castelo Branco (Portugal)

Fontes de Gracia, S; García, C.; Garriga, A,; Pérez-Llantada, Mª̊.; Sarriá, E. (2007) Diseños de Investigación en Psicología. Madrid: UNED.

Fredricks, S.L. y Eccles, J.S. (2006). Extracurricular involvement and adolescent adjustment: impacto $\mathrm{f}$ duration, number of activites, and breadth of participation. Applied Development Science, 10(3), 132-146.

Fundació Jaume Bofill (2006). Debat familiar. ¿Què opinen les famílies sobre les activitats eductives fora de l'horari escolar?. Ayuntament de Barcelona.

Fundació Jaume Bofill (2009). Diagnosis de les activitats eductives fora de l'horar escolar a Barcelona. Institut d'educació. Ayuntament de Barcelona.

García Preciados, A.V. (2001). La educación física y el deporte: evolución de los intereses de la población extremeña. Tesis doctoral. Universidad de Extremadura.

García Ferrando, M. (1991). Los españoles y el deporte (1980-1990): un análisis sociológico. Madrid: ICEFD, CSD y Ministerio de Educación y Ciencia.

García Ferrando, M. (1993). Tiempo libre y actividades deportivas de la juventud en España. Madrid: $\mathrm{M}^{\circ}$ Asuntos Sociales e Instituto de la Juventud.

García Ferrando, M. (1996a). Cambios en los hábitos deportivos de los españoles. Temas para el debate, 23: 43-46.
García Ferrando, M. (1996b). La encuesta. En: M. García Ferrando, J. Ibáñez y F. Alvira: El análisis de la realidad social. Métodos y técnicas de investigación. Madrid: Alianza Universidad Textos.

García Ferrando, M. (2001). Los españoles y el deporte. Prácticas y comportamientos en la última década del siglo XX: encuestas sobre los hábitos deportivos de los españoles. Madrid: CSD.

García Ferrando, M. (2006). Veinticinco años de análisis del comportamiento deportivo de la población española (1980-2005). Revista Internacional $d d e$ Sociología, 44, 15-38.

García Preciados, A.V. (2001). La educación física y el deporte: evolución de los intereses de la población extremeña. Tesis Doctoral. Universidad de Extremadura.

Gavarry, O., Giacomoni, M., Bernard, T., Seymat, M. \& Falgairette, G. (2003). Habitual Physical Activity in children and adolescents during school and free days. Medicine and Science in sports and exercise, 35(3), 525-531.

González Pozuelo, F. (1999). Escolares extremeños-as del 2000. Un estudio sociológico para la igualdad de género. Consejería de Cultura. Junta de Extremadura.

Gilman, R., Meyers, J. y Pérez, L. (2004). Structured extracurricular activities among adolescents: finding and implications for school psychologists. Psychology in the Schools, 4(1), 31-41

Guerrero, A. (2000). Evolución del deporte en edad escolar en España, antecedentes, situación actual. En I Congreso Nacional de Deporte en edad escolar, (pp.21-62). Dos Hermanas (Sevilla). Exmo Ayuntamiento de Dos Hermanas.

Hernández, J.L. y Velázquez, R. (1996). La actividad física y deportiva extraescolar en los centros educativos. Madrid: MEC.

Hernández, J.L. y Velázquez, R. (coords.) (2007). La Educación Física, los estilos de vida y los adolescentes: cómo son, cómo se ven, qué saben y qué opinan. Estudio de la población escolar y propuestas de actuación. Barcelona: Graò.

Hernández Sampieri, R; Fernández Collado, C y Baptista Lucio, P (2006). Metodología de la investigación.4a edición. Mc Graw Hill. México D.F. p.282. y 291.

Hidalgo Rivas, R.F. (2005). El impacto de las actividades extraescolares en ciencia y tecnología. En A. Martín; I. Trilles y G. Zamarrón (Eds.): Universidad y comunicación social de la ciencia (pp.177-197). Granada: Editorial Universidad de Granada.

Instituto Nacional de Calidad y Evaluación (2001). Actividades del alumno fuera del horario escolar. Actividades extraescolares. INCE (11). Disponible en www.ince.mec.es [Consulta 2009, 3 de septiembre].

Luengo Vaquero, C. (2007). Actividad físico-deportiva extraescolar en alumnos de primaria Revista Internacional de Medicina y Ciencias de la Actividad Física y el Deporte 7(27), 174-184. Disponible en http://cdeporte.rediris.es/revista/revista27/ artactividadf41c.htm [Consulta 2009, 20 de Julio].

Mendoza, R., Sagrera, M. y Batista, J.M. (1994). Conductas de los escolares españoles relacionados con la salud. Madrid: CSIC.

Molinuevo, B. (2008) Actividades Extraescolares y salud mental: estudio de su relación en población escolar de Primaria. Tesis Doctoral. Universitat Autónoma de Barcelona.

Molinuevo, B. (2009) El temps fora de l'horari lectiu: els recursos a la llar, les activitats i els agents educatius. En R. Torrubia y E. Doval (coord.). Família i Educació a Catalunya (pp. 122-195). Fundació Jaume Bofill.

Mollá Serrano, M. (2007). La influencia de las actividades Extraescolares en los Hábitos deportivos de los Escolares. Revista Internacional de Medicina y Ciencias de la Actividad Física y el Deporte vol. 7(27) pp. 241-252. Disponible en http:// cdeporte.rediris.es/revista/revista27/artinfluencia41f.htm [Consulta 2009, 20 de Julio].

Nuviala A., Ruiz F., Ruiz, F. y García, ME. (2003) Tiempo libre, ocio y actvidad física en los adolescentes. La influencia de los padres. RETOS. Nuevas tendencias en Educación física y Recreación. 6, 13-20.

Nuviala A., Ruiz F., Murguía, D. Fernández, A. Ruiz, F. y García, ME.(2009) Typologies of occupation of leisure-time of Spanish adolescents. The case of the partcicipants in physical activities organized. Journal of Human Sport and science, 4(1), 29-39.

Pérez-Díaz, V., Rodríguez, J.C. y Sánchez, L. (2001). La familia espanyola davant l'edcucaió dels seus fills. Colección Estudios Sociales, 5. Barcelona: Fundación la Caixa

Pettit, G.S., Laird, R.D., Bates, J.E. y Dodge, K.A. (1997). Patterns of after school care in middle childhood: risk factors and developmental outcomes. Merrill-Palmer Quaterly, 43, 515-538. En Molinuevo, B. (2008) Actividades Extraescolares y salud mental: estudio de su relación en población escolar de Primaria. Tesis Doctoral. Universitat Autónoma de Barcelona.

Ponce de León, A. (1998). Análisis de la E.F. escolar desde la perspectiva de una educación para el tiempo libre. Apunts (51) 23-34.

Sallis, J.F. (2000). Age-related decline in physical activity: a synthesis of human and animal studies. Med Sci Sports Exerc, 32, 1598-1600.

Santos, M. y Sicilia, A. (1998). Actividades físicas extraescolares: una propueste alternativa. Barcelona: INDE.

Tercedor, P.; Delgado, M.; Pérez, I.; Chillón, P.; González-Groos, M., $\quad$ Montero, A.; Moreno, L.A.; De Rufino-Rivas, P. y Torralba, C. (2003). $\quad$ A v e n a Group. Physical activity level in spanish adolescents. The avena study. Actas de II Congreso Mundial de Ciencias de la Actividad Física y el Deporte. Deporte y Calidad de Vida. Granada.

Trilla J. y García I. (2004). Infancia y tiempo libre organizado. En J.Gómez-Granell, J. García, Ripio-Millet y C. Panchón (coords). Infancia y familia: realidades y tendencias. Barcelona: Ariel.

Trilla, J. y Ríos, O. (2005). Les activítats extraescolars: diferències i desigualtats. En C.Gómez-Granell et al., Infància, familia i canvi social a Catalunya. Barcelona: Consorci Institut d’Infància i Mon Urbá.

Varela, L. (2006). Evaluación de programas extraescolares: importancia del nivel de participación en las percepciones sobre el tiempo libre, r e n d i m i e n t o académico y habilidad deportiva de los participantes. Revista galego-portuguesa de psicoloxía e educación, 11-12(3), 207-219. 